\title{
The Electrocardiogram in the Differential Diagnosis of Cardiologic Conditions Related to the COVID-19 Pandemic
}

Bruna Predabon' ${ }^{1}$ Arthur Zanfrilli Marques Souza1, Gustavo Henrique Sumnienski Bertoldi', Rafaela Louise Sales ${ }^{2}$, Karila Scarduelli Luciano ${ }^{2}$, Rafael de March Ronsoni ${ }^{1,2, *}$

\section{ORCID IDs}

Predabon B (D) https://orcid.org/0000-0002-3877-4461

Sales RL (D) https://orcid.org/0000-0002-3831-3016

Souza AZM (D) https://orcid.org/0000-0002-3757-6922

Luciano KS (1) https://orcid.org/0000-0002-5210-8414

Bertoldi GHS (D) https://orcid.org/0000-0001-6347-3137

Ronsoni RM (D) https://orcid.org/0000-0001-7135-9844

\begin{abstract}
The World Health Organization (WHO) declared Sars-CoV infection and COVID-19 as a pandemic and global emergency. In addition to viral pneumonia and the severe acute respiratory syndrome (Sars), the heart is affected in some patients due to the detection of biomarkers and reported cases of myocarditis and pericarditis. Therefore, the knowledge of electrocardiogram presentation of these actual infections can guide the choice for the best treatment and can help to reduce misdiagnosis, mainly the acute myocardial infarction, which is the main differential diagnosis.
\end{abstract}

KEYWORDS: Myocarditis; COVID-19; Electrocardiographic.

1. Universidade da Região de Joinville - Departmento de Medicina - Joinville (SC), Brazil.

2. Hospital Regional Hans Dieter Schmidt - Departmento de Cardiologia - Joinville (SC), Brazil.

*Correspondonding author: rafaelronsoni@gmail.com

Received: Jun 12, 2020 | Accepted: Aug 12, 2020 


\section{INTRODUCTION}

The Sars-CoV-2 outbreak initiated at Wuhan, China, is a novel kind of coronavirus with similarities when compared to its proteomics with Sars-CoV, and its main way to get access to the cells is via the angiotensin converting enzyme $2^{1-3}$. Its main clinical features are fever (89.1\%), cough (72.2\%), and fatigue (42.5\%); severe acute respiratory syndrome (Sars) affects $14.8 \%$ of the patients with $96.6 \%$ presenting with computed tomography (CT) abnormalities ${ }^{2}$.

Sars-CoV-2 infection is associated with a variety of pro-inflammatory mediators that may play important roles in the pathophysiology of cardiac complications. In single centers studies, cardiac injury was observed between 10-19\% of hospitalized patients with COVID-19 and was associated with higher risk of in-hospital mortality ${ }^{4,5}$. Collaborating with these data, some recent cases showed up myocarditis and pericarditis in patients with Sars-COV-2 infection, even in patients without respiratory symptoms ${ }^{6-8}$.

Thus, the diagnosis of pericarditis caused by Sars-CoV-2 has clinical and prognostic significance, and the role of electrocardiogram (ECG) has been fundamental. Alterations in the PR segment can facilitate the differential diagnosis, mainly with myocardial infarction with ST-segment elevation, in addition to reducing invasive procedures and unnecessary pharmacological treatment. It is important to remember that deviations in the PR segment are often ignored in the clinical and hospital environment, however, up to $82 \%$ of patients with acute pericarditis had changes in the PR segment and rarely in other clinical situations. 9 . Our objective was to describe clinical situations related to changes in the PR segment and the differential diagnoses, with emphasis on myopericarditis due to COVID-19 pandemic myopericarditis.

\section{DEFINITIONS}

Myocarditis refers to an inflammatory process of the heart muscle and results in positive biomarkers, electrocardiographic changes, and histological findings including inflammatory infiltrate with degeneration and necrosis in non-ischemic regions. The term pericarditis refers to isolated inflammation of the membranes around the heart, but in the presence of electrocardiographic changes it means an extension to the epicardium, once the parietal pericardium is electrically inert. The term myopericarditis is used when the inflammatory process occurs in both structures (pericardium and myocardium) $)^{9-11}$

The changes on PR segment and P-wave can occur in pericarditis due to epicardium extension. The alterations can also be involved in other clinical situations, though with different mechanisms, such as: sympathetic overstimulation, atrial dilatation or hypertrophy, and intra-atrial blocks. The effect of this superficial myocarditis in the thin-walled atria leads to exaggerated atrial $\mathrm{T}$ waves and resulting $\mathrm{PR}$ segment depression ${ }^{12}$.

Typical electrocardiogram alterations can occur in $60-90 \%$ of pericarditis cases. Stage 1 electrocardiographic manifestations are easily confused with ST-elevation myocardial infarction (STEMI), being the stage in which the changes in the PR segment are described and can be of great value for the differential diagnosis between the two situations ${ }^{13}$. Quickly, the 4 phases of pericarditis in relation to the ST-segment and T wave are: first elevation of the ST-segment, which can occur in all leads, except for V1 and aVR; second ST-segment returns to baseline and flat T wave; third, the $\mathrm{T}$ wave reverses and depression of the ST-segment may occur; fourth, normalization occurs in weeks or months ${ }^{14}$. The main clinical situations associated with changes in the PR segment are mentioned below.

\section{Early repolarization with ST-segment changes (ERSTE)}

The American Heart Association defines early repolarization all types of ST-elevation (inferior end/or antero-lateral leads) in the absence of chest pain. It must always be accompanied by a description that should include whether there is ST elevation, terminal QRS notch, terminal QRS slurring or J-point elevation ${ }^{15}$. 
The classic electrocardiographic ECG manifestation of stage I acute pericarditis is diffuse ST elevation and PR depression with ST depression in the lead aVR. But this pattern cannot be considered pathognomonic, since Birnbaum Y. et al described four patients with early repolarization with ST-segment changes (ERSTE). All patients had ST elevation in lead II with either ST elevation or isoelectric ST in lead I with concomitant ST depression in aVR. Therefore, the set of electrocardiographic changes must be analyzed rather than just the classic electrocardiographic pattern for the diagnosis of pericarditis (Fig. 1$)^{16}$.

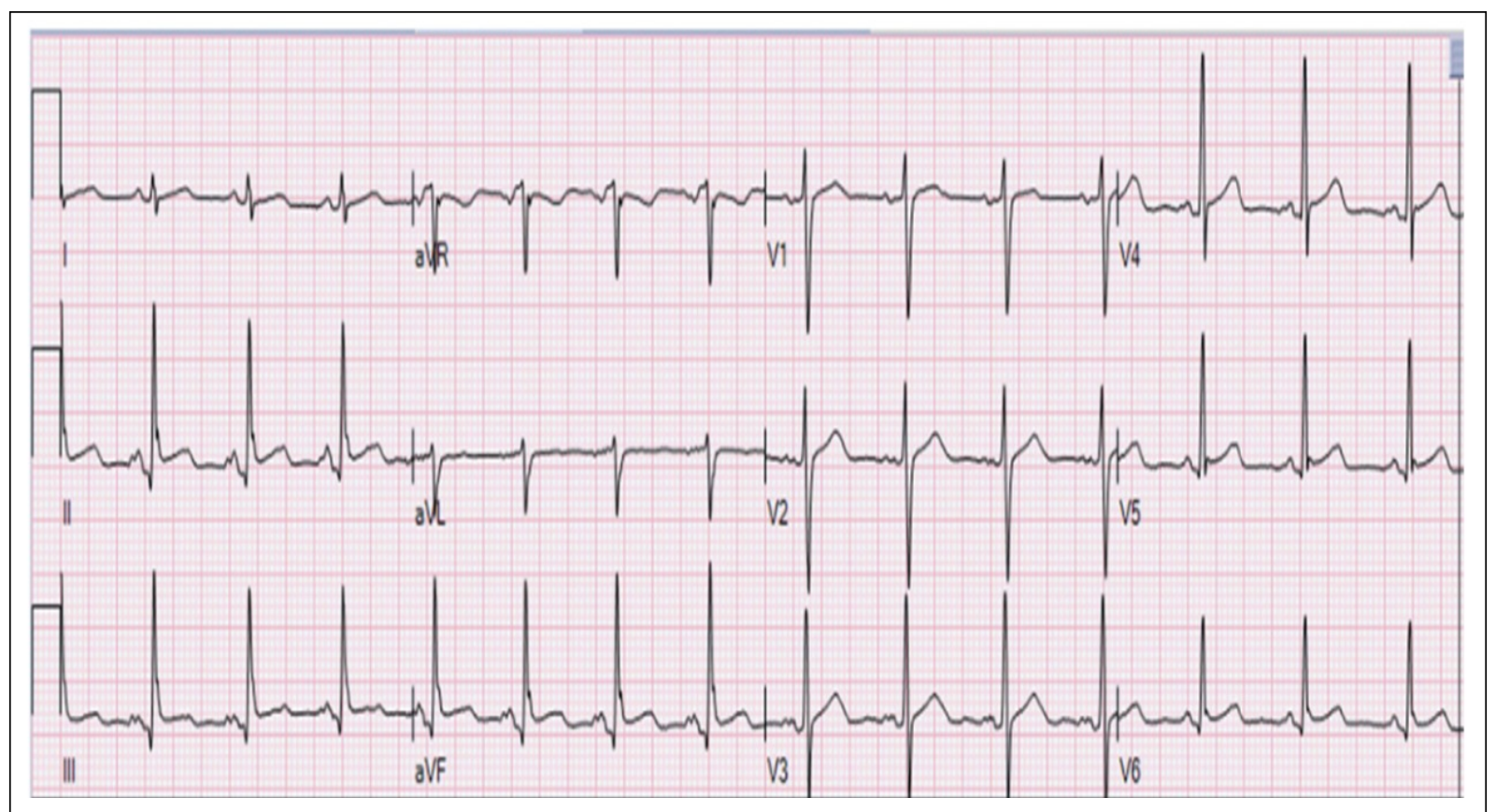

Figure 1. Case for ERSTE - SR associated with P wave with the presence of notching; presence of PR depression in the inferior wall and between V3-6; presence of PR elevation in aVR; presence of end_QRS slurring in the inferior wall; presence of end-QRS notching in V4-6; presence of ST depression in aVR. (adapted from Birnbaum et al. ${ }^{17}$ ).

In this clinical situation, alteration of the PR segment may be fundamental for the differential diagnosis with pericarditis. The classic work of Spodick D. confers that the diagnosis of pericarditis is favored by PR-segment deviations simultaneously in limb and precordial leads, in patients with ERSTE ${ }^{17}$. However, when the diagnosis is not based on the previous data, it is necessary to evaluate the most frequent ST-segment manifestations in each situation, listed below:

- Lead V1:

- ST-segment depression: most often in pericarditis $(29 \text { vs. } 5 \%)^{16,17}$.

- Lead V6:

- Isoelectric segment: most often in ERSTE (21 vs. 2\%).

- ST-segment elevation ( $\geq 0.5 \mathrm{~mm})$ : present in $44 \%$ of ERSTE cases $^{16,17}$.

- Leads DII, III, avF (inferior):

- ST-segment elevation of the internal wall in greater amplitude than the ST-segment elevation of the lateral wall, occurs more frequently in ERST $^{16,17}$.

- ST-T segment vector:

- Pericarditis is between 0 and 30 degrees in $52 \%$ of the cases.

- ERSTE is between 70 and 90 degrees in $55 \%$ of them ${ }^{16,17}$.

-We suggest not using the Imazio index (ST-segment amplitude/T-wave amplitude $>0.24$ suggests pericarditis) due to low sensitivity in this patient subgroup ${ }^{16,17}$. 


\section{Atrial Infarction}

Necropsy studies reveal a prevalence of 0.7 to $42 \%$ of atrial infarction in the context of acute coronary syndrome, rarely occurring in isolation and the vast majority affecting the right atrium in relation to the left (98 vs. 19\%). The atria are supplied by the superior ramus ostium cava, originating in $60 \%$ of the time in the right coronary artery and $40 \%$ in the circumflex artery. P wave changes in the context of STEMI may occur as a result of ischemia, increased sympathetic activity, atrial dilation, and intra-atrial block ${ }^{13,18}$.

The traditional criteria for atrial infarction are:

- Major criteria: PR segment elevation greater than $0.5 \mathrm{~mm}$ in leads V3 and V6 with depression in lead V1 and V2.PR segment elevation of more than $0.5 \mathrm{~mm}$ in lead I with depressions in leads II and III. PR segment depression greater than $1.5 \mathrm{~mm}$ in precordial leads, and $1.2 \mathrm{~mm}$ depression in leads I, II, and III, associated with any atrial arrhythmia.

- Minor criteria: abnormal $\mathrm{P}$ wave morphology (flattening of the $\mathrm{P}$ wave in "M"or "W" and notch presence)13,18.

Currently, they are criticized for 2 reasons: their publication was not validated afterward and a cohort of STEMI published with 666 individuals where none of the patients fulfilled any of the criteria described above $\mathrm{e}^{13,18}$.

The main described changes associated with atrial infarction in STEMI cohorts are (Fig. 2):

- $\mathrm{P}$ wave with abnormal morphology in $35 \%$ of patients;

- Deviations from the PR segment ( $\geq 0.5 \mathrm{~mm}$ elevation and $\geq 0.8 \mathrm{~mm}$ depression) are identified in $31 \%$ of patients and are strongly suggestive of atrial infarction ${ }^{13,18}$;

- In STEMI, depression of the PR segment in the inferior wall is a prognostic criterion $(\geq 1.2 \mathrm{~mm})$ associated with atrioventricular block, supraventricular arrhythmias, and rupture of the free wall with a consequent increase in mortality in 1 year ${ }^{19,20}$.

The topographic correlation is not universally accepted, but some authors suggest this correlation:

- Right atrium: elevation of the PR segment in DII and DIII leads with reciprocal depression in DI.

- Left atrium: elevation of the PR segment in DI lead and reciprocal depression in V2-4 and DII-III ${ }^{13}$.

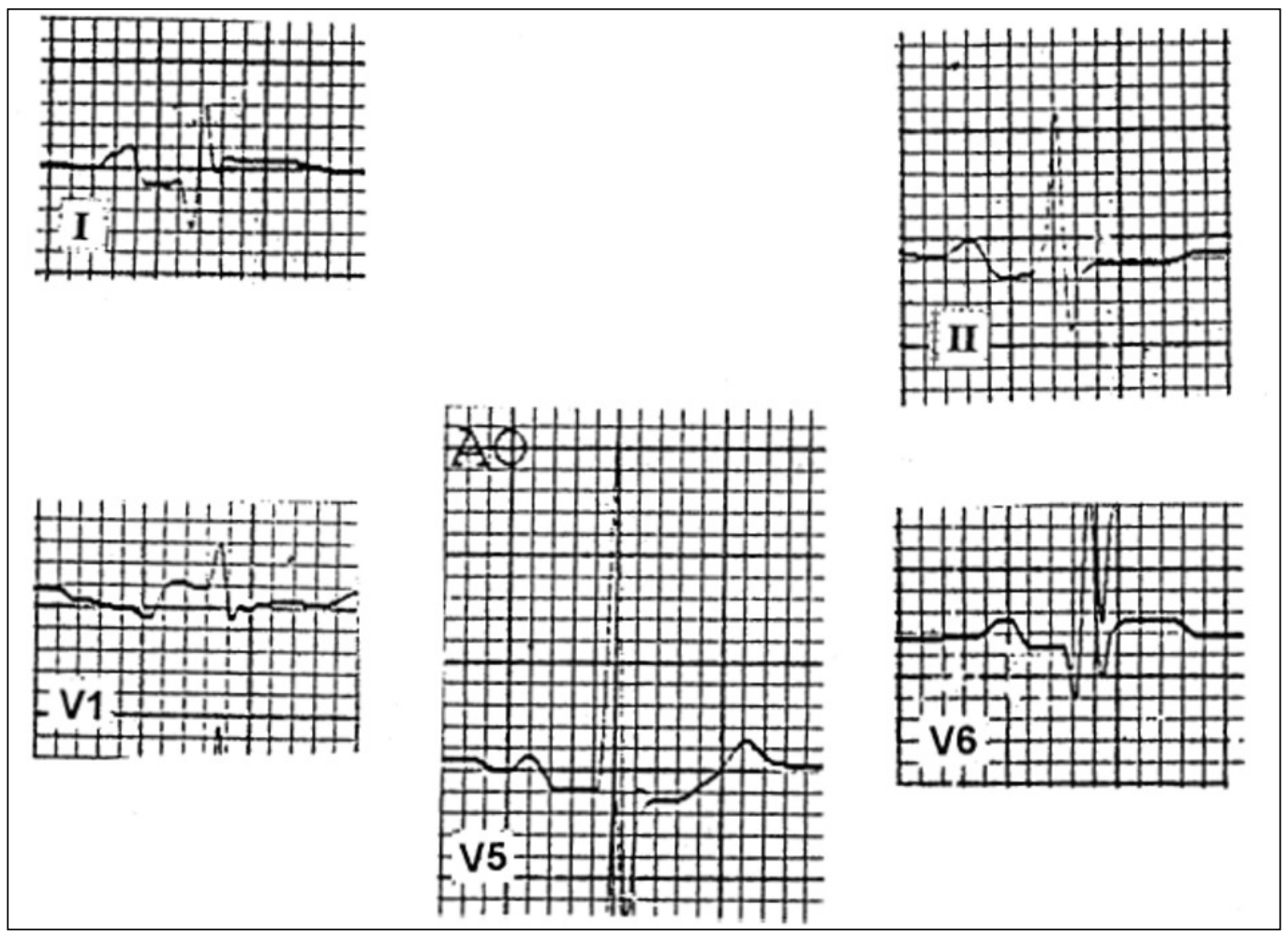

Figure 2. Presence of changes in the PR segment in the period of atrial infarction. 


\section{Takotsubo cardiomyopathy}

PR-segment depression is found in up to $63 \%$ of cases of Takotsubo cardiomyopathy (TC) and is much more prevalent than STEMI (62 vs. 3\%). The pathophysiology is associated with increased adrenergic activity, namely catecholaminergic storm, which is a feature of the disease and generates PR-segment depression though with an isoelectric Ja point. The impact of adrenergic activity on action potentials in cardiac myocytes causes ascending ST-segment elevation and depressed J point. In many cases, the amplitude of the ST-segment elevation is reduced in postmenopausal women due to a reduction in repolarizing ion channels sensitive to catecholamines caused by a decrease in estrogen concentrations ${ }^{21}$. Several TC cases are associated with emotional stress, and the present COVID-19 pandemic can be considered a triggering factor.

Previous studies reported that PR depression occurs in both pericarditis and TC. Diagnostic criteria to assist the differential diagnosis when associated with ST-segment elevation in the inferior wall are suggested ${ }^{21-23}$.

- ST-segment depression in lead aVL results in a diagnosis of STEMI, excluding the differential diagnosis between STEMI and TC, and STEMI and pericarditis.

The main electrocardiographic criteria to differentiate TC and STEMI, especially in postmenopausal women with chest pain, which is the main epidemiological TC group, were (Fig. 3) ${ }^{21}$ :

- Association of ST-segment elevation $\leq 0.2 \mathrm{mV}$ with PR-segment depression.

- TC diagnosis with sensitivity of $52 \%$, specificity of $100 \%$, positive predictive value of $100 \%$, and negative predictive value of $67 \%$.

Care should be taken with using only ST-segment depression in aVR to suggest TC instead of STEMI, as this finding does not provide sufficient accuracy for the electrocardiographic differentiation of these situations ${ }^{22-24}$.

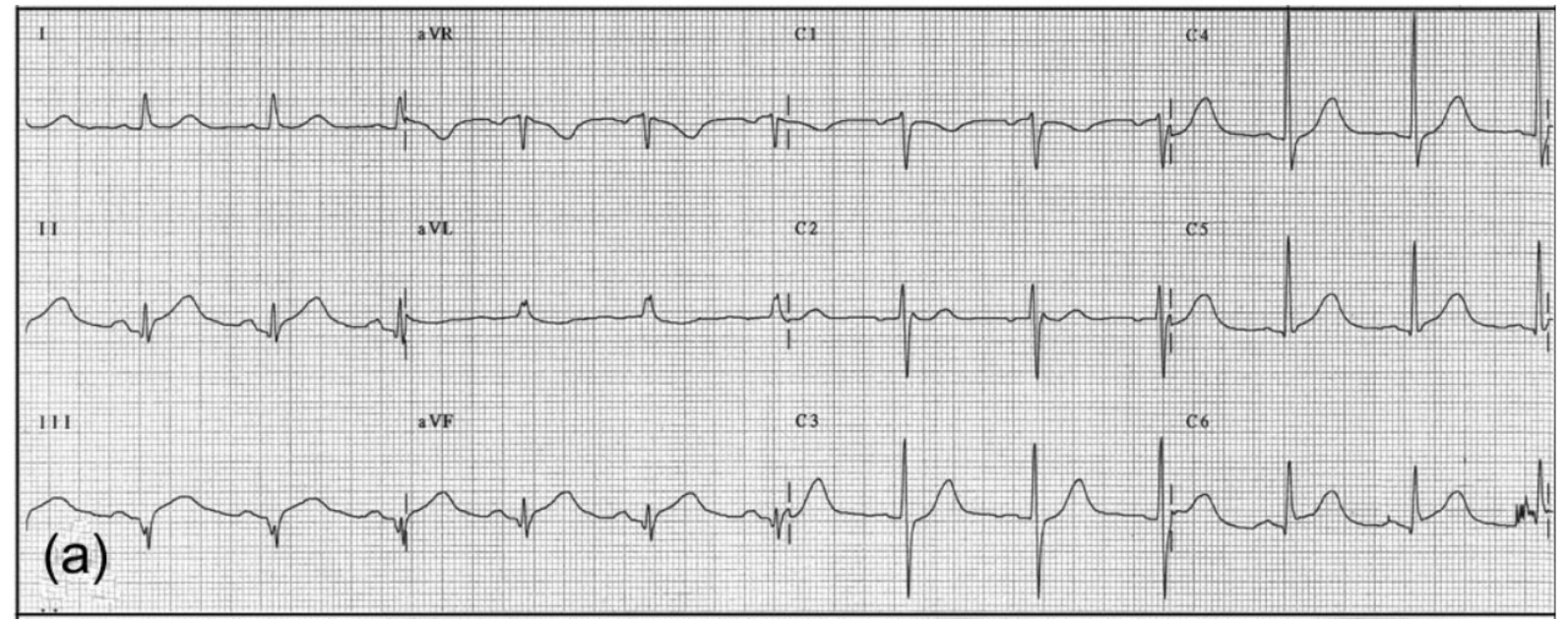

Figure 3. PR-segment depression in the inferior wall and ST-segment elevation $(\leq 0.2 \mathrm{mV})$ in the inferior wall and V5-6 in an 81-year-old woman ${ }^{23}$.

\section{ST-elevation myocardial infarction}

The differential diagnosis of myopericarditis and STEMI in acute coronary syndromes is of utmost importance, since a misdiagnosis can result in harmful consequences to patients ${ }^{22}$. In the presence of coronary occlusion, an outward shift in repolarizing currents occurs (decrease in $\mathrm{Na}+$ or $\mathrm{Ca}++$ and increase in $\mathrm{K}+$ ion currents) and leads to a loss of the action potential dome in the epicardium, but not in the endocardium ${ }^{23}$. Generally, in myopericarditis, ST-segment alterations characterized by an elevation of the J point (usually $\leq 5 \mathrm{~mm}$ ) with a concave shape and terminal QRS notching or slurring with diffuse involvement (limb and precordial leads) - may be present except for V1 and aVR, where a reciprocal change is found (Fig. 4). In contrast, in STEMI, elevation of the J point with a convex shape or upsloping flat is observed in contiguous leads related to transmural ischemia and reciprocal ST-segment depression ${ }^{10,19,22}$. 


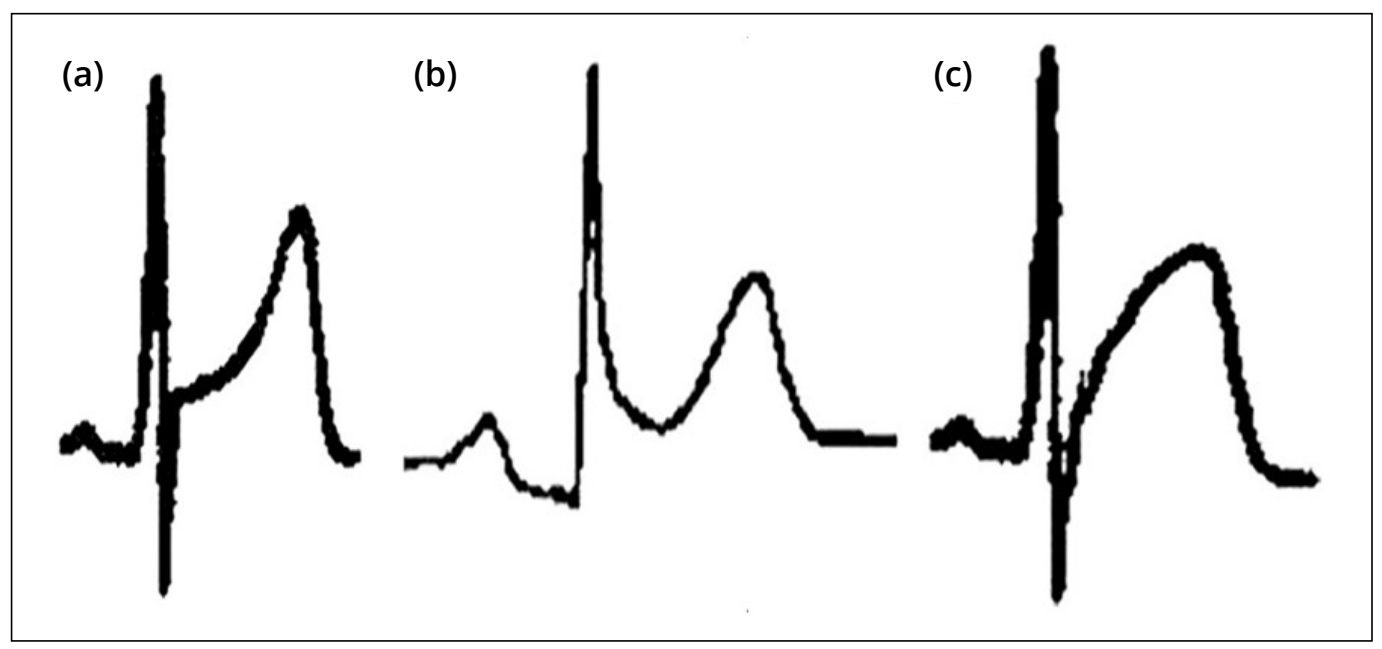

Figure 4. (a) ST-segment elevation in ERSTE. (b) ST-segment elevation with PR segment depression of myopericarditis. (b) STEMI (adapted from Pollak and Brady, 2012).

The main electrocardiographic changes between the two clinical situations will be mentioned in the differential diagnosis. A cutoff point of $0.5 \mathrm{~mm}$ is used in the quantitative analysis of the deviation of the PR segment to define it as pathological ${ }^{10}$.

- Classical electrocardiographic pattern of myopericarditis but not pathognomonic ${ }^{19}$ :

- Lead aVR

- Greater PR-segment elevation and deeper ST-segment depression in lead aVR (PR-segment deviation $\geq 0.5 \mathrm{~mm}$ );

- Multiple leads affected by ST-segment elevation; and

- Reciprocal ST-segment depression identified in aVR and V1;

- Diagnostic criterion that supports the diagnosis of pericarditis with sensitivity of $85.9 \%$ and specificity of $85.3 \%$ (presence of all items) ${ }^{19}$ :

- PR-segment elevation in lead aVR;

- ST-segment depression (J-point $\geq 0.05 \mathrm{mV}$ ) in lead aVR;

$\bullet \geq 7$ leads with ST-segment elevation;

$\bullet \leq 1$ lead with ST-segment depression; and

- $\geq 0$ leads with PR-segment depression.

Diagnostic criterion that supports the diagnosis of pericarditis with positive predictive value of $96.7 \%$ and negative predictive value of $90 \%$.

- Association of PR-segment depression in both precordial and limbs leads, with possible exceptions in aVR and V1 due to reciprocal changes ${ }^{10,19,25}$ :

- PR-segment depression may occur in 9\% of STEMI, but chest limbs are likely not affected ${ }^{22}$; and

- In pericarditis, changes in the PR segment are usually observed in II, aVR, aVF, and V4-6 and more prominently in II and V5-6 ${ }^{10}$.

- Diagnostic criterion that supports the diagnosis of pericarditis using QRS duration and Qtc interval in leads with maximum ST-segment elevation compared to leads with an isoelectric ST-segment:

- In pericarditis, QRS duration and Qtc interval do not change in those leads;

- In STEMI, QRS duration increases (cutoff, $70 \mathrm{~ms}$ ) and the Qt interval decreases (cutoff of dispersion, $63 \mathrm{~ms}$ ) comparing the two leads ${ }^{26}$; and

- Zhan et al. recently published an interesting case of atrial infarction where the application of this criterion favored the diagnosis of ischemic origin in PR-segment changes accompanied by STEMI ${ }^{19}$.

- Other electrocardiographic diagnostic criteria

- ST-segment depression in lead aVL in the presence of inferior STEMI. 
- It favors the diagnosis of STEMI with high sensitivity and specificity, since the lead with the highest prevalence of ST-segment depression was DII in $55.9 \%$ of the cases of myopericarditis and that with the one with the lowest prevalence was aVL in $2.9 \%$ of cases $^{22-24}$.

- Lee's criterion (Fig. 5).

- Leads V2 and V3: Absence of waves J and S in leads V2 and V3 suggests STEMI, with 100\% specificity for anterior descending artery occlusion ${ }^{27}$.

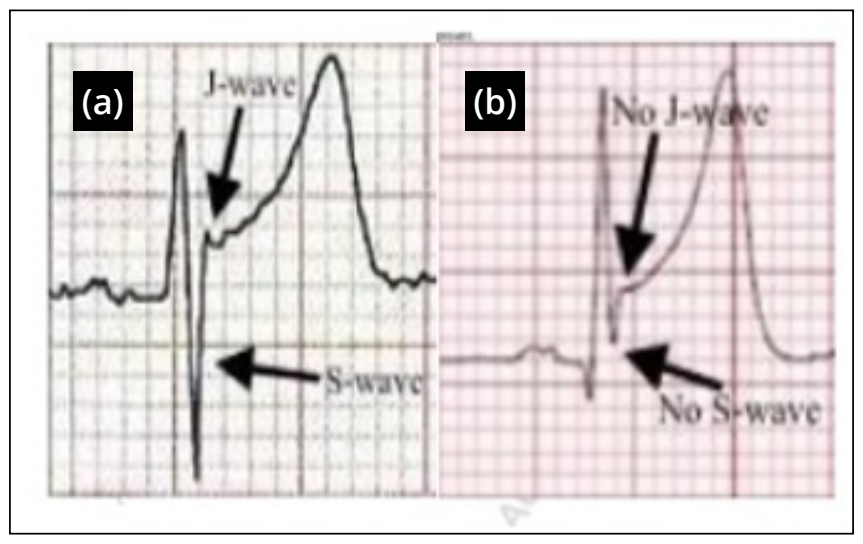

Figure 5. (a) ERSTE pattern with the presence of J and S waves. (b) Lee's criterion: absence of J and S waves, predictor of STEMI (adapted from Lee et al., 2016).

- Spodick's sign (Fig. 6).

- It is present in up to $80 \%$ of patients with pericarditis, which comprises a descending baseline between ST-segment elevation and PR-segment depression of the next beat ${ }^{28}$.

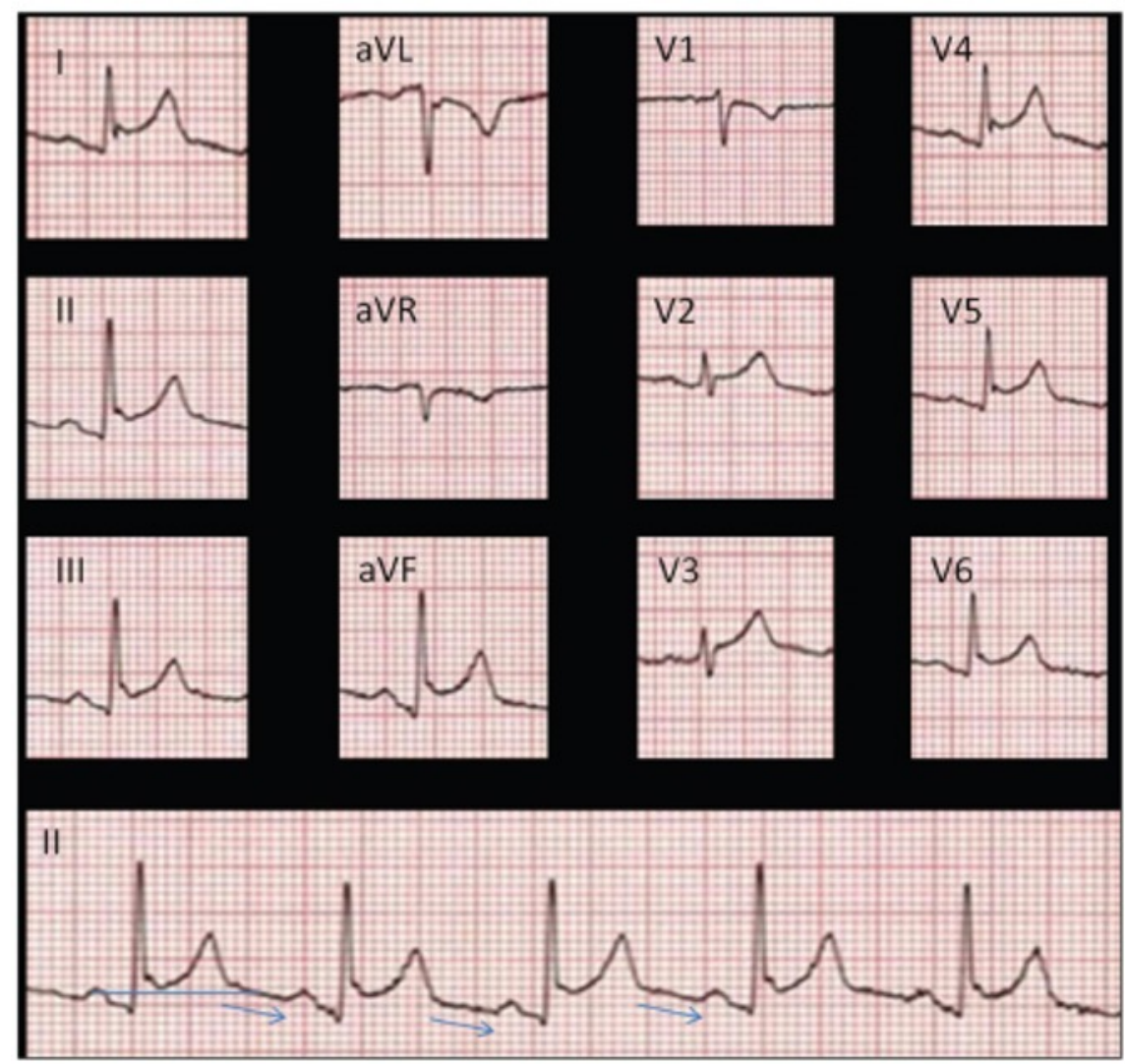

Figure 6. Presence of the Spodick's sign. Arrows show descending baselines between ST-segment elevation and PR-segment depression of the next beat. 
- Imazio's index.

- Amplitude of the supra ST-segment/amplitude to the peak of the $\mathrm{T}$ wave $>0.24$ suggests pericarditis in leads $\mathrm{DI}, \mathrm{V} 4, \mathrm{~V} 5$, and $\mathrm{V} 6^{15,16}$.

- Progression with the presence of pathological Q waves associated with STEMI14.

Again, hospitalization is important in this situation since most patients with pericarditis, even with involvement of multiple leads, will be clinically stable, unlike cases of extensive STEMI, which will have clinical repercussions ${ }^{11}$. We strongly believe that PR-segment and QT interval measurements are vital in differential diagnosis ${ }^{9}, 19$. A diagnostic error of STEMI in cases of myopericarditis may lead to unnecessary invasive interventions or even fibrinolysis, which is potentially associated with mortality in myopericarditis ${ }^{11}$.

\section{CONCLUSION}

With the first described cases of myopericarditis in the present COVID-19 pandemic, it is essential to have a wide knowledge of the electrocardiographic changes associated with this clinical condition, which are often neglected in a widely available and low-cost examination. Through the publication of electrocardiographic features, we hope to promote a rapid and accurate diagnosis and adequate treatment for this clinical situation, thus avoiding measures associated with possible clinical complications that are mainly related to an erroneous diagnosis of STEMI.

\section{REFERENCES}

1. Huang C, Wang Y, Li X, Ren L, Zhao J, Hu Y, et al. Clinical features of patients infected with 2019 novel coronavirus in Wuhan, China. Lancet. 2020;395(10223):497-506. https://doi.org/10.1016/S0140-6736(20)30183-5

2. Xu J, Zhao S, Teng T, Abdalla AE, Zhu W, Xie L, et al. Systematic Comparison of Two Animal-to-Human Transmitted Human Coronaviruses: Sars-CoV-2 and Sars-CoV. Viruses. 2020;12(2):244. https://doi.org/10.3390/v12020244

3. Wan Y, Shang J, Graham R, Baric RS, Li F. Receptor Recognition by the Novel Coronavirus from Wuhan: an Analysis Based on DecadeLong Structural Studies of Sars Coronavirus. J Virol. 2020;94(7):e00127-20. https://doi.org/10.1128/JVI.00127-20

4. Sun P, Qie S, Liu Z, Ren J, Li K, Xi J. Clinical characteristics of hospitalized patients with Sars-Cov-2 infection: a single arm metaanalysis. J Med Virol. 2020;92(6):612-7. https://doi.org/10.1002/jmv.25735

5. Chen C, Zhou Y, Wang DW. Sars-CoV-2: a potential novel etiology of fulminant myocarditis. Herz. 2020;45(3):230-2. https://doi. org/10.1007/s00059-020-04909-Z

6. Shi S, Qin M, Shen B, Cai Y, Liu T, Yang F, et al. Association of Cardiac Injury With Mortality in Hospitalized Patients With COVID-19 in Wuhan, China. JAMA Cardiol. 2020;5(7):802-10. https://doi.org/10.1001/jamacardio.2020.0950

7. Inciardi RM, Lupi L, Zaccone G, Italia L, Raffo M, Tomasoni D, et al. Cardiac Involvement in a Patient With Coronavirus Disease 2019 (COVID-19). JAMA Cardiol. 2020;5(7):819-24. https://doi.org/10.1001/jamacardio.2020.1096

8. Zeng J-H, Liu Y-X, Yuan J, Wang F-X, Wu W-B, Li J-X, et al. First Case of COVID-19 Infection with Fulminant Myocarditis Complication: Case Report and Insights. Preprints. 2020. https://doi.org/10.20944/preprints202003.0180.v1

9. Hua A, O'Gallagher K, Sado D, Byrne J. Life-threatening cardiac tamponade complicating myo-pericarditis in COVID-19. Eur Heart J. 2020;41(22):2130. https://doi.org/10.1093/eurheartj/ehaa253

10. Celik T, Ozturk C, Balta S, lyisoy A. The role of combined electrocardiogram criteria in differential diagnosis of acute pericarditis: PR segment and QT interval. Am J Emerg Med. 2016;34(7):1309. https://doi.org/10.1016/j.ajem.2016.04.013

11. Buttà C, Zappia L, Laterra G, Roberto M. Diagnostic and prognostic role of electrocardiogram in acute myocarditis: A comprehensive review. Annals of Noninvasive Electrocardiology. 2020;25(3). https://doi.org/10.1111/anec.12726

12. Pollak P, Brady W. Electrocardiographic Patterns Mimicking ST Segment Elevation Myocardial Infarction. Cardiol Clin. 2012;30(4):60115. https://doi.org/10.1016/j.ccl.2012.07.012 
13. Spodick DH. Diagnostic Electrocardiographic Sequences in Acute Pericarditis. Significance of PR Segment and PR Vector Changes. Circulation. 1973;48(3):575-80. https://10.1161/01.cir.48.3.575

14. Lu MLR, De Venecia T, Patnaik S, Figueredo VM. Atrial myocardial infarction: A tale of the forgotten chamber. Int J Cardiol. 2016;202:904-9. https://doi.org/10.1016/j.ijcard.2015.10.070

15. Spodick DH. Electrocardiogram in acute pericarditis. Distributions of morphological and axial changes by stages. Am J Cardiol. 1974;33(4):470-4. https://doi.org/10.1016/0002-9149(74)90603-1

16. Patton KK, Ellinor PT, Ezekowitz M, Kowey P, Lubitz SA, Perez M, et al. Electrocardiographic Early Repolarization: A Scientific Statement from the American Heart Association. Circulation. 2016;133(15):1520-9. https://doi.org/10.1161/CIR.0000000000000388

17. Birnbaum Y, Riera ARP, Nikus K. PR depression with multi-lead ST elevation and ST depression in aVR: Is it always acute pericarditis? J Electrocardiol. 2019;54:13-7. https://doi.org/10.1016/j.jelectrocard.2019.01.085

18. Spodick DH. Differential Characteristics of the Electrocardiogram in Early Repolarization and Acute Pericarditis. N Engl J Med. 1976;295(10):523-6. https://doi.org/10.1056/NEJM197609022951002

19. Lu MLR, Nwakile C, Bhalla V, De Venecia T, Shah M, Figueredo VM. Prognostic significance of abnormal P wave morphology and PR-segment displacement after ST-elevation myocardial infarction. Int J Cardiol. 2015;197:216-21. https://doi.org/10.1016/j. ijcard.2015.06.055

20. Zhan Z-Q, Nikus K, Birnbaum Y. PR depression with multilead ST elevation and ST depression in aVR by left circumflex artery occlusion: How to differentiate from acute pericarditis. Ann Noninvasive Electrocardiol. 2020;early view. https://doi.org/10.1111/ anec. 12752

21. Jim M-H, Siu C-W, Chan AO-O, Chan RH-W, Lee SW-L, Lau C-P. Prognostic implications of PR-segment depression in inferior leads in acute inferior myocardial infarction. Clin Cardiol. 2006;29(8):363-8. https://doi.org/10.1002/clc.4960290809

22. Lindow T, Pahlm O, Khoshnood A, Nyman I, Manna D, Engblom H, et al. Electrocardiographic changes in the differentiation of ischemic and non-ischemic ST elevation. Scand Cardiovasc J. 2020;54(2):100-7. https://doi;org/10.1080/14017431.2019.1705383

23. Zorzi A, Baritussio A, ElMaghawry M, Siciliano M, Migliore F, Marra MP, et al. Differential diagnosis at admission between Takotsubo cardiomyopathy and acute apical-anterior myocardial infarction in postmenopausal women. Eur Hear J Acute Cardiovasc Care. 2016;5(4):298-307. https://doi.org/10.1177/2048872615585515

24. Porela P, Kytö V, Nikus K, Eskola M, Airaksinen KEJ. PR Depression Is Useful in the Differential Diagnosis of Myopericarditis and ST Elevation Myocardial Infarction. Ann Noninvasive Electrocardiol. 2012;17(2):141-5. https://doi.org/10.1111/j.1542474X.2012.00489.x

25. Adler C, Halbach M, Adler J, Michels G, Reuter H. Differenzialdiagnostische Bedeutung der PR-Strecke in der EKG-Interpretation. Medizinische Klin - Intensivmed und Notfallmedizin. 2018;113(1):50-2. https://doi.org/10.1007/s00063-017-0364-z

26. Rossello X, Wiegerinck RF, Alguersuari J, Bardají A, Worner F, Sutil M, et al. New Electrocardiographic Criteria to Differentiate Acute Pericarditis and Myocardial Infarction. Am J Med. 2014;127(3):233-9. https://doi.org/10.1016/j.amjmed.2013.11.006

27. Lee DH, Walsh B, Smith SW. Terminal QRS distortion is present in anterior myocardial infarction but absent in early repolarization. Am J Emerg Med. 2016;34(11):2182-5. https://doi.org/10.1016/j.ajem.2016.08.053

28. Makaryus JN, Makaryus AN, Boal B. Spodick's Sign. Am J Med. 2008;121(8):693-4. https://doi.org/10.1016/j.amjmed.2008.01.020 